\title{
Barter trade improves message delivery in opportunistic networks
}

\author{
Levente Buttyán ${ }^{\mathrm{a}}$ László Dóra ${ }^{\mathrm{a}, *}$ Márk Félegyházi ${ }^{\mathrm{b}}$ István Vajda $^{\mathrm{a}}$ \\ ${ }^{a}$ Laboratory of Cryptography and Systems Security (CrySyS), \\ Budapest University of Technology and Economics, Hungary \\ b University of California, Berkeley, USA
}

\begin{abstract}
In opportunistic networks, selfish nodes can exploit the services provided by other nodes by downloading messages that interest them, but refusing to store and distribute messages for the benefit of other nodes. We propose a mechanism to discourage selfish behavior based on the principles of barter. We develop a game-theoretic model in which we show that the proposed approach indeed stimulates cooperation of the nodes. The results show that, in practical scenarios, the message delivery rate considerably increases, if the mobile nodes follow the Nash Equilibrium strategy in the proposed mechanism compared to the data dissemination protocol when no encouraging mechanism is present.
\end{abstract}

Key words: Opportunistic networks, Game-theory

PACS: 02.50.Le

\section{Introduction}

An opportunistic network is a mobile ad hoc network where the transfer of messages from their source to their destination is performed by the intermediate mobile nodes in a store-carry-and-forward manner. This means that the intermediate nodes carry the messages and pass them on to other intermediate nodes when they have a connection (e.g., when they are in vicinity).

Such networks can complement traditional personal wireless communications systems, such as cellular networks, in applications where local information needs to be distributed to a set of nearby destinations based on their interest in the information.

As a motivating example, let us consider a touristic city, such as Rome or Paris, where it would be beneficial for the tourists to be able to share information concerning the various touristic sights. A possible solution would be to set up an on-line bulletin board where tourists can post messages of potential interest for other tourists. However, this solution needs a service provider that runs the bulletin board service, and each tourist must have wireless Internet access for posting and downloading messages. The business

\footnotetext{
* Corresponding author.

Email addresses: buttyan@crysys.hu (Levente Buttyán), dora@crysys.hu (László Dóra), felegyha@eecs.berkeley.edu (Márk Félegyházi), vajda@crysys.hu (István Vajda).
}

model behind this solution would likely require the tourists to pay for both the service usage and the network access.

An alternative solution could benefit from the proliferation of Bluetooth capable personal devices such as mobile phones, PDAs, and MP3 players. These devices can communicate with each other when they are in vicinity even without any user intervention. Touristic information can then be distributed in a store-carry-and-forward manner by using these devices and by exploiting the mobility of the tourists themselves. This would result in a city-wide opportunistic network.

A potential problem in opportunistic and in delaytolerant personal wireless networks is that the quality of the service provided by the system heavily depends on the users' willingness to cooperate. In particular, the users may act selfishly meaning that they download messages from other users that are interesting for them, but they deny storing and distributing messages for the benefit of other users. As shown in [1], if the majority of the users behave selfishly, then the message delivery rate decreases considerably and the quality of service provided by the network decreases accordingly.

The problems identified in [1] are the motivation for proposing a mechanism that encourages the users to carry other users' messages even if they are not directly interested in those messages. Our proposed mechanism is based on the principles of barter: the users trade in messages and 
a user can download a message from another user if he/she can give a message in return. We expect that it is worth for the users collecting messages even if they are not interested in them to exchange them later for messages that they are interested in. Thus, the messages are expected to disseminate faster in the network.

We analyze our proposed solution using game-theoretic techniques. We show that it is worth for the users collecting and disseminating messages even if they are not interested in them, which means that our approach indeed discourages selfishness. The results show that, in practical scenarios, the message delivery rate considerably increases, if the mobile nodes follow the Nash Equilibrium strategy in the proposed mechanism compared to the data dissemination protocol when no encouraging mechanism is present.

This paper is a considerably extended version of our previous work [2]. The differences between the two papers are manyfold. We have rebuilt the system and the game model to be more realistic, and we extended the simulation sets, too. In this paper, we present new contributions and a more detailed analysis of the results.

The remainder of the paper is organized as follows. In Section 2, we analyze the system without any incentives and determine the scenarios where stimulating mechanism should be introduced. In the same section, we introduce the system model that is used to analyze the system with and without encouragement. We describe our barter based approach, and we also extend the system model with the barter mechanism in Section 3. For the analysis of the effects of selfish behavior on the system augmented with the barter mechanism, we introduce a game-theoretic model in Section 4. In Section 5, we show and interpret the results of the barter game. We summarize the related work in Section 6. Finally, we conclude this paper in Section 7.

\section{System analysis}

In this section, we introduce our system model, which is general enough to represent different applications, and it is particularly well adapted for the example touristic scenario described above. Because of the complexity of the model, we use simulations instead of analytical tools. We show that there are scenarios where the message delivery has large latency because the mobile nodes are selfish in a sense that they only store and forward messages that they are directly interested in. The aim of the analysis is twofold: 1) to prove that an incentive is required in the network to increase the message delivery rate and decrease the message delivery latency, and 2) to give a reference with which we can compare our subsequent solution.

\subsection{System model}

In our model, the users are placed in an arbitrary field. They own devices that have capabilities to communicate with other devices within their radio range. We consider the case when the devices communicate via wireless links, however, or analysis can be extended to wired communication too. The used wireless technology can be Bluetooth, Wi-fi or any suitable wireless techniques. The messages are generated and disseminated among the devices/users in the considered system, but each user is interested only in a small subset of the messages. The dissemination process is based on the store-carry-and-forward principle. A user and her device together is the mobile node, and we assume that the message destination has no impact on the user's movement.

Each message has a type for each mobile node. For simplicity, we distinguish only two types: primary messages and secondary messages. A message is a primary message for a given mobile node, if the mobile node is interested in the content of the message and secondary if the mobile node is not. Note that a message may have different types for different mobile nodes, as different mobile nodes are interested in different contents.

These messages are generated by special nodes which we call message nodes. In our system model the time is slotted, and the message nodes generate new messages with a fixed average rate: $\varrho$ messages per time step. The message nodes are static and each one stores only the most recently generated message, which can be downloaded at the cost of communication by any mobile node that passes by the message node.

A message has two main properties: the first one is the popularity attribute and the second one is the discounting characteristic. The popularity attribute $0<\zeta \leq 1$ describes the probability that a randomly taken mobile node is interested in the message. We assume that message nodes do not generate irrelevant messages, hence we consider $\zeta>0$.

Each message has some value for each mobile node. The value of a message is determined by its age. For simplicity, we assume that primary messages of the same age have the same value for the mobile nodes. Without loss of generality, we assume that the value of a primary message at the time of its generation is one unit, and this is discounted in time, because messages lose their value over time. This is usually the case in the applications that opportunistic networks are envisioned for. The discounting characteristic is described with a function: $\delta(t)$. The discounting function determines the value of the messages over time. Obviously, it is difficult or impossible to find a discounting function which suits to each application. Therefore, we defined three different monotonely decreasing discounting functions. We express these function in Eqs. (1)-(3) and we plot them in Figure 1. In the first case, the message value decreases linearly, in the second case, the messages devaluate exponentially, and in the last case, the messages lose their value suddenly, similarly to a step function. 


$$
\begin{aligned}
& \delta_{0}(t)= \begin{cases}1-\frac{t}{500} & \text { if } t<500 \\
0 & \text { else }\end{cases} \\
& \delta_{1}(t)=0.995^{t} \\
& \delta_{2}(t)=1-\frac{1}{1+1000 \cdot\left(1-\frac{1}{20}\right)^{t}}
\end{aligned}
$$

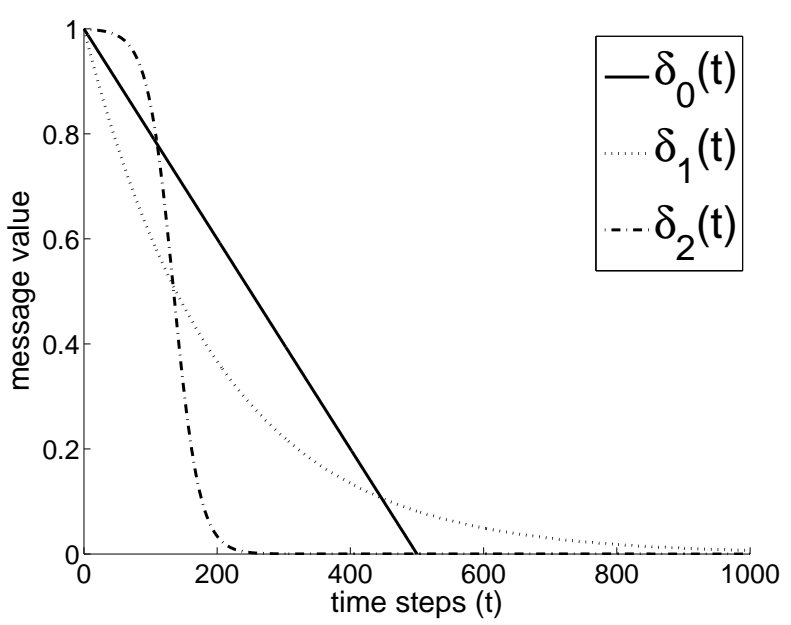

Fig. 1. Devaluation of primary messages over time

When two mobile nodes get in the vicinity of each other, they interact in the following way:

(i) The mobile nodes exchange the list of the messages that they carry. The exchanged lists contain only the short descriptions of the messages (including their time of generation) rather than the messages themselves.

(ii) Each mobile node $u$ removes from the list $L_{v}^{(0)}$ received from $v$ the messages that are not primary for node $u$, and the ones that $u$ already stores in memory getting the list $L_{v}^{(1)}$.

(iii) Each mobile node $u$ determines the value of the messages listed in $L_{v}^{(1)}$ based on their ages. Then, each mobile node orders the messages contained in $L_{v}^{(1)}$ by their value in descending order. The resulting ordered list $L_{v}^{(2)}$ is the list of messages that $u$ wishes to download from $v$.

(iv) Mobile nodes $u$ and $v$ download messages from each other following the lists $L_{v}^{(2)}$ and $L_{u}^{(2)}$, respectively, until they move out from each other's radio range.

Connections can be interrupted because the mobile nodes are mobile and they leave the radio range of the other party. Therefore, in our model, the mobile nodes are not able to exchange as many messages as they want but at maximum one message per time step. Hereby, we assume that a message exchange is either completed in the time step or not started at all. This limited exchange capability is called the implicit cost of the exchange, because there is no guarantee that the nodes can download all the messages that they want from the other party.

In our system model, there is no other costs. In a scenario that we imagine the communication cost is negligible as the battery of the personal devices can be recharged easily day by day. The storage cost has two aspects: 1) The messages need storage space and storage constraint may limit the number of stored messages. This limitation is not significant as the storage space required for storing the data downloaded by using wireless technology is less than the memories offer, nowadays. 2) The time needed to determine which messages and in what order the nodes want to download increases polynomially with the number of message stored by the other party. To control this, the mobile nodes delete the valueless messages, thus, they delete the messages from the memory whose value goes below a certain threshold $D, 0<D<1$.

To measure the message delivery rate and delivery latency, we define a formula for the goodput (see Eqs. (4) and (5)). The notation is the following considering mobile node $i$ :

- $m_{i}^{t}$ is the message that mobile node $i$ downloaded in time step $t$

- $T_{m}$ is the time step when message $m$ was generated;

$-\delta$ is the discounting function described above;

- $v_{i}(t)$ is the gain that mobile node $i$ gets in time step $t$, and it is defined as follows:

$$
v_{i}(t)=\delta\left(t-T_{m_{i}^{t}}\right)
$$

Let $M_{i}^{P}(t)$ denote set of messages that were generated until time $t$ and are primary for node $i$. The cardinality of $M_{i}^{P}(t)$ describes the maximum value that node $i$ can obtain until time $t$ as the value of each message is 1 at the moment of the generation. As shown in (5), the goodput $\left(0 \leq G_{i}(t) \leq 1\right)$ for mobile node $i$ is the sum of the gains in each time step normalized with the value that node $i$ could obtain in an ideal case.

$$
G_{i}(t)=\frac{\sum_{\tau=0}^{t} v_{i}(\tau)}{\left|M_{i}^{P}(t)\right|}
$$

Note that the goodput is time and mobile node specific. However, the distribution of $G_{i}$ is same for each mobile node $i$ if all the mobile nodes behave in the same way. The goodput may vary over time, however we will show in Appendix A that the value of the goodput converges to a steady-state value. Therefore, we will consider the goodput, denoted by $G_{i}$, of each mobile node $i$ in the steady-state conditions.

$$
G_{i}=\lim _{t \rightarrow \infty} G_{i}(t)
$$

\subsection{Simulations}

In our simulations, the fixed-number of mobile nodes move in discrete time steps according to one of the two mobility models: the Restricted Random Waypoint (RRW) and Simulation of Urban MObility (SUMO, [3]) model.

In the restricted random waypoint model, 300 mobile nodes move on a field of size $20 \times 20$ unit initially placed 
uniformly at random. On the field, there are some special points chosen at random; these are called meeting points. Each mobile node selects a meeting point randomly, and moves towards this meeting point along a straight line with a fixed speed. When the meeting point is reached, the mobile node stops and stays for randomly chosen time (10 time steps on average). Then, it chooses another meeting point and begins to move again. The nodes that happen to be at the same meeting point in the same time step are paired randomly and these pairs are able to download one message from each other in the above described way.

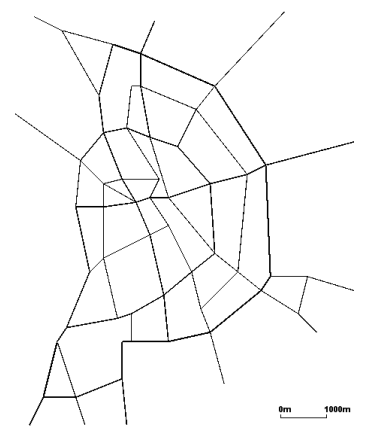

Fig. 2. Simplified map of Budapest used in SUMO mobility model

SUMO is an open source, realistic road traffic simulator. 300 vehicles (mobile nodes) start their movement from a randomly chosen place at a randomly chosen time and they follow the traffic rules moving towards their destination also chosen at random in a predefined map. We implemented a simplified map of Budapest, Hungary with 60 intersections (including the dead ends) in SUMO as shown in Figure 2 and the vehicles move on this map. In each edge, there is a speed limit specified calculated automatically by the SUMO (in most cases $35 \mathrm{~m} /$ time step and sometimes $40 \mathrm{~m} /$ time step). The vehicles accelerate, move constantly at the highest speed, slow down and stop depending on the traffic. The nodes can communicate with each other when they stop in the intersections similarly to the meeting points in the restricted random waypoint model. The vehicles leave the meeting point as soon as the traffic admits.

In Figure 3, we compare the two considered mobility model with respect to the duration of getting from a meeting point to another neighboring one. Two meeting points $(A$ and $B$ ) are neighbors if a mobile node can go from $A$ to $B$ and back without stopping at any other intermediate meeting points. Note that in the case of restricted random waypoint model any two meeting points are neighbors, but in the case of SUMO, only those meeting points that are linked in Figure 2. In Figure 3(a) and 3(b), the histogram of the time steps needed to reach a meeting point is shown. In the case of the SUMO, the duration was specified considering the maximum speed of the cars. As a comparison we can state that in the restricted random waypoint mobility model the mobile nodes communicate with each other more frequently than in the case of SUMO.
Recall that in our system model, the messages are injected into the network by message nodes that are static. In the restricted random waypoint model, the message nodes reside in the meeting points, whereas in SUMO the message nodes are placed in each intersection.

As we have already described each message has a popularity value $\zeta$. When a message node generates a new message in the simulation, it determines which mobile node is interested in it according to the popularity value. Thus, the message node sets the message to primary with probability $\zeta$ for each mobile node. All the message nodes together generate one new message per time step on average both in case of SUMO and restricted random waypoint model.

In each simulations, all the messages have the same discounting characteristic, one of the function described in Subsection 2.1 (see Eqs. (1)-(3)).

We determined the length (number of time steps) of the simulation in an empirical way by taking into account that the goodput have to reach the steady-state goodput. When we run the simulations for 3000 time steps, the average goodput have not changed considerably for 1000 time steps in the analyzed simulations. Therefore we run all simulations for 3000 time steps.

We summarize the simulation parameters in Table 1.

Table 1

Parameter values of the simulations

\begin{tabular}{|c|c|c|}
\hline Parameter & RRW & SUMO \\
\hline Simulation length (time steps) & \multicolumn{2}{|c|}{3000} \\
\hline Number of mobile nodes & \multicolumn{2}{|c|}{300} \\
\hline Number of meeting/cross points & 100 & 60 \\
\hline Number of message nodes & 100 & 60 \\
\hline Message generation rate $\varrho$ & 0.01 & 0.0166 \\
\hline Simulation area & $20 \times 20$ unit & see Fig. 2 \\
\hline Velocity (unit/time step) & 1 & \multirow{2}{*}{$\begin{array}{c}\text { induced by } \\
\text { SUMO }\end{array}$} \\
\hline Probability of leaving a meeting point & 0.1 & \\
\hline Threshold for message erase $D$ & \multicolumn{2}{|c|}{0.05} \\
\hline
\end{tabular}

We varied some of the parameters to study their effect on the results. As described above during simulation runs we used different functions for message devaluation. Besides this, for the sake of simplicity, we assumed that during a simulation the messages are generated with one predefined popularity attribute $\zeta$, but we executed more simulations with different $\zeta$ values. Recall that $0<\zeta \leq 1$. To reduce the complexity of our simulations, we use the following values of $\zeta: \zeta=0.05,0.2,0.4,0.6,0.8,1$.

The main objective of these initial simulations is to prove that an incentive is required to increase the message delivery rate and to decrease the message delivery latency. Therefore, we run two kinds of simulations for every scenario: 1) one to get the goodput when the nodes behave selfishly, and 2) another one to get an upperbound for the goodput. In the former case, the mobile nodes strictly follow the protocol introduced in Section 2.1. This protocol corresponds to selfish behavior, because the mobile nodes 


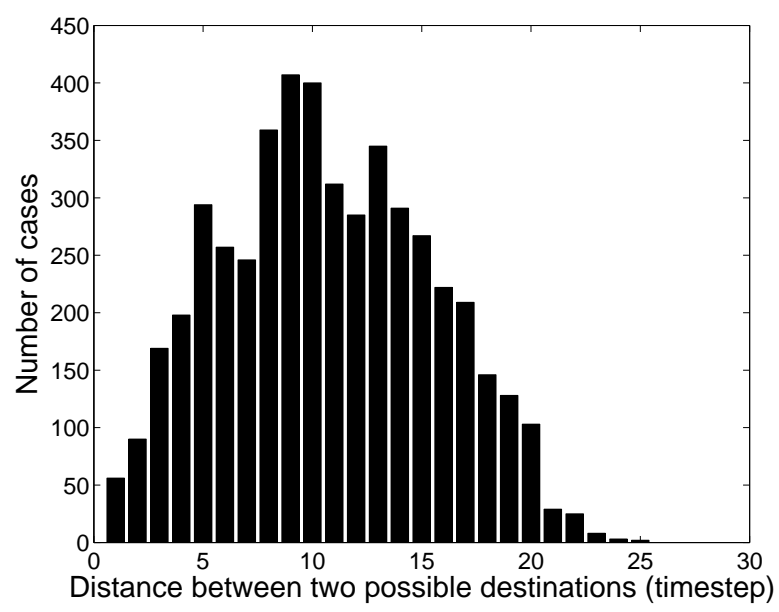

(a) RRW

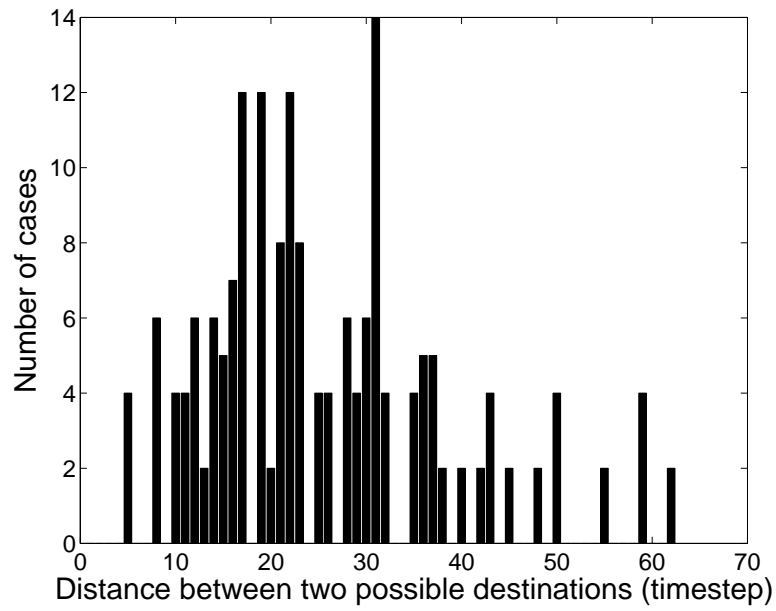

(b) SUMO

Fig. 3. Histogram of the duration of getting from a meeting point to another neighboring one

download only those messages in which they are interested. To get an upperbound for goodput, the mobile nodes download all the new messages that they find in the memory of the connected node in one time step, both the primary and secondary ones. Clearly, this upperbound is different from the theoretical maximum of 1 , because the value of a message decreases before reaching an interested mobile node, if reaches it at all.

As we have already stated, the distribution of the goodput achieved by the mobile nodes is the same. Therefore, we determine the goodput of the network by getting the average goodput of all the nodes.

\subsection{Motivation}

Results in the case of the restricted random waypoint model and SUMO can be seen in the Figures 4(a) and 4(b), respectively. In these figures, we show simulations where the discounting function is linear $\left(\delta_{0}\right)$, because the results show minor changes with other message devaluations.

In these figures, the goodput of the network is plotted against the popularity attribute value of the messages. To remind the reader, in the simulations in each parameter set, the messages have the same popularity value. The solid line shows an upperbound for the goodput and the line with dashes and dots shows the goodput of the network in the selfish case, when the mobile nodes do not download secondary messages. We present the $95 \%$ confidence intervals at each simulation points.

There are significant differences between the two mobility models. In the case of the restricted random waypoint model (shown in Figure 4(a)) the goodput is much higher than the one in the SUMO mobility model (shown in Figure 4(b)). This difference has two reasons:

- In SUMO mobility model, the traffic is higher at the central meeting points than in the suburb as it is the case in all the cities. Where the traffic is low, the mobile nodes can quickly bypass the message nodes. For this reason, the messages generated there may be deleted before passing to any mobile node. Recall that a message node can store only one message. Therefore, the message node overwrites a message if a new one is generated.

- As Figure 3 shows, in the case of SUMO mobility model, the distances between the meeting points are longer than in the case of restricted random waypoint model. Recall that mobile nodes are able to exchange messages only while they do not move. Furthermore, in the SUMO, the mobile nodes can bypass quicker the meeting points than in the restricted random waypoint model. All in all, the mobile nodes have less opportunity to exchange messages in the case of SUMO.

When the mobile nodes behave selfishly the popularity value has a large impact on the goodput. The more mobile nodes are interested in a message, the more nodes download the message even if all the mobile nodes are selfish. The more mobile nodes download a message, the higher is the probability that a mobile node will meet one who has already downloaded the message. We call this the selfish carrier effect and it can be seen in the Figure 4(b), but not clearly in the Figure 4(a). There, the goodput increases with the increasing popularity until a specific value, but then the goodput decreases.

The reason for the decrease of the goodput while the popularity increases is the following: The goodput is a ratio as Eq. (5) shows. As one can see, the denominator (maximum value) can increase to infinity. While the numerator (obtained value) has an upperbound (even if it is difficult to determine in a concrete parameter set), because the nodes are able to exchange only one message in each time step. Thus, if the number of the interested messages increases, but the obtained value reached its upper limit, then the goodput decreases considerably.

To conclude the motivation section, we can state that the goodput is affected by two mainly independent, but opposite effects: the selfish carrier effect and the implicit cost. When the value of the popularity attribute is 1 the good- 


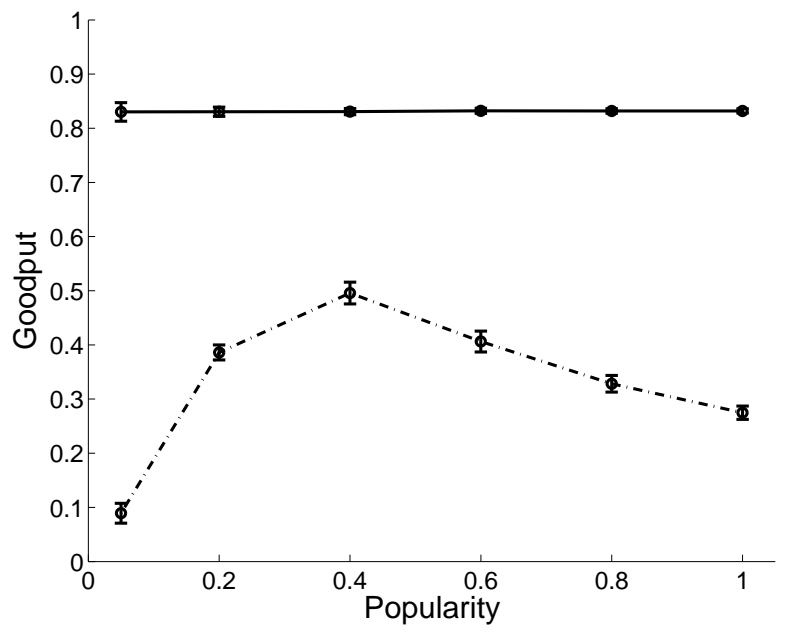

(a) RRW

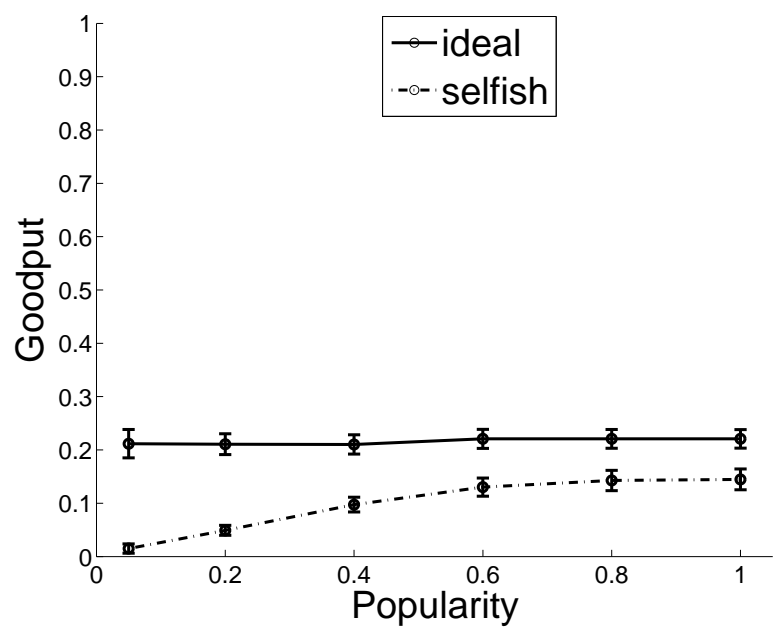

(b) SUMO

Fig. 4. Goodput without encouragement: The steady state goodput plotted against the popularity value of generated messages when discounting function is linear $\left(\delta_{0}\right)$ and the mobile nodes move according to restricted random waypoint and SUMO mobility model in Figure $a$ and $b$, respectively. The bars show the $95 \%$ confidenance interval.

put is affected mainly by the implicit cost, whereas when the popularity value is near to 0 it is affected clearly by the selfish carriers. The implicit cost comes from a property of the system model, while the selfish carrier effect comes from the selfishness of the mobile nodes. Therefore, we can state that an incentive is required to compensate the selfish carrier effect which mainly affects the goodput of the network when the popularity value of the generated messages is low.

\section{Our approach}

Our approach to stimulate the cooperation of mobile nodes is based on the principles of barter. More specifically, as mentioned above when two nearby mobile nodes establish a connection, they first send the description of the messages that they currently store to each other, and then they agree on which subset of the messages they want to download from each other. In order to ensure fairness, the selected subsets must have the same size, and the messages are exchanged in a message-by-message manner, in preference order. If any party cheats, the exchange can be disrupted, and the honest party does not suffer any major disadvantage (i.e., the number of messages downloaded by the honest party is at most one less than the number of messages downloaded by the misbehaving party).

Note that it is entirely up to the mobile nodes to decide which messages they want to download from each other. They may behave selfishly by downloading only those messages that are of primary interest for them. However, selfish behavior may not be beneficial in the long run. In particular, the idea is that a message that is not interesting for a mobile node $A$ may be interesting for another mobile node $B$, and $A$ may use it to obtain a message from $B$ that is indeed interesting for $A$. In other words, the messages that are secondary for a mobile node still represent a barter value for the mobile node, and hence, it may be worth downloading and carrying them. Thus, the messages can be viewed as an investment to get new primary messages later.

Recall that the selfish mobile nodes ignore the secondary messages when they selected the messages to download in the message exchange protocol introduced in Section 2.1. However, when the messages are exchanged according to the principles of barter, as it is mentioned above, it is worth downloading and carrying secondary messages too, even if the mobile nodes are selfish (we will show that this statement holds). But, the mobile nodes have to compare the value of primary to the value of the secondary messages when they select which messages and in what order they want to download from the connected party.

Recall that there is no direct benefit of downloading a secondary message. It is worth to download to exchange later for primary ones. According to this, the value of the secondary messages is considered only when a node sorts the messages for downloading from another node. The value of a secondary message at the time of its generation depends on how the mobile node values secondary messages with respect to primary messages. The secondary value is discounted in the same way as primary messages. In other words, if for a mobile node, secondary messages are worth $S P$ units for some $0 \leq S P \leq 1$ at the time of their generation, then the value of a secondary message after $t$ time units is $S P \cdot \delta(t) . S P$ is called secondary/primary ratio. We have to emphasize that if $S P_{u}=0$ then the mobile node $u$ does not download any secondary messages.

Note that in general, the value of a secondary message cannot be larger than the value of a primary message of the same age (i.e., $S P \leq 1$ ), because the primary message has the same barter value as the secondary message, and in addition, the mobile node is interested in its content. However a specific secondary message which is more fresh than a specific primary message may have higher value and 
it can be exchanged for primary messages later, which will have higher gain all together.

We adapt the message exchange protocol according to the barter based approach in the following way:

(i) The mobile nodes exchange the list of the messages that they carry.

(ii) Each mobile node $u$ removes from the list $L_{v}^{(0)}$ received from $v$ the messages that $u$ already stores in memory, and thereby obtains the list $L_{v}^{(1)}$.

(iii) Each mobile node $u$ determines the value of the messages listed in $L_{v}^{(1)}$ based on their types, their ages, and the secondary/primary ratio $S P_{u}$ as described above. The list obtained in this way is denoted by $L_{v}^{(2)}$

(iv) Each mobile node $u$ orders the messages contained in $L_{v}^{(2)}$ by their value in descending order. The resulting ordered list $L_{v}^{(3)}$ is the list of messages that $u$ wishes to download from $v$.

(v) The nodes exchange at most $\ell=\min \left(\left|L_{u}^{(2)}\right|,\left|L_{v}^{(2)}\right|\right)$ messages from the beginning of their lists on a message-by-message manner, where $|L|$ denotes the length of the list $L$. Thus, the number of exchanged messages is determined by the length of the shorter list or the duration of the connection.

We assume that the mobile nodes offer all their valid and only valid messages to download. It is not worth for any nodes to hide messages from other mobile nodes, because it may decrease the number of messages that the mobile node is allowed to download from other mobile nodes. In addition, we assume that a mechanism is present in the system that prevents injecting fake messages. This is important, because greedy nodes can increase the number of messages that they can offer by injecting fake messages.

In order to show that the latter assumption is feasible, we sketch the operation of two mechanisms that would prevent the injection of fake messages:

- One prevention mechanism can be based on digital signatures. The mobile nodes are allowed to exchange only those messages that have a valid digital signature. The digital signatures are added to the messages by an authority. This authority can be represented by the message nodes and in that case, the message nodes are responsible not just for generating the messages but certifying them, too. Although, this solution filters out the fake messages it may not be applicable in some application.

- Another mechanism for preventing injections of fake messages can be based on reputation mechanism. The users can define a threshold and they download only messages whose reputation value is higher than the threshold and the users can evaluate the messages or the services which generates the messages themselves. The evaluation messages may be distributed among the mobile nodes. Note that this reputation mechanism is not related to the mobile nodes' willingness of the message distribution, but it refers to the quality of the message contents. This kind of reputation mechanism can complement our barter mech- anism.

The purpose of our analysis later in this paper is to verify whether the barter based approach increases the goodput or not.

\section{Game model}

We model our proposed mechanism as a game to analyze the behavior of the mobile nodes using game-theory [47]. Our objective is to prove that the network can reach high goodput using barter mechanism even if selfish mobile nodes are present.

We define a non-cooperative game $G=\left[P,\left\{S_{i}\right\},\left\{\pi_{i}\right\}\right]$, called barter game. $P$ is the set of the players, $S_{i}$ denotes the strategy space of player $i \in P$, and $\pi_{i}$ represents the payoff function of each player $i$. To be more precise, $\pi_{i}$ is the simplified notation of $\pi_{i}\left(s_{0}, s_{1}, \ldots, s_{|P|}\right)$, because the payoff of each player depends on the strategy played by the other players. This can also be denoted by $\pi_{i}\left(s_{i}, s_{-i}\right)$ emphasizing the strategy of player $i$, where $s_{-i}$ is the strategy profile of all the players except for player $i$.

In the barter game, the players $(P)$ are the mobile nodes, and hence in the rest of this paper, we will use the same notation for players as for mobile nodes. The strategy of each player is its secondary/primary ratio $\left(S P_{i} \in S_{i}=\right.$ $[0,1])$. The players do not change their strategies during the game. The players choose their strategies in a way to maximize their goodput. Hence, the steady-state goodput is the payoff of the barter game for player $i$.

$$
\pi_{i}=G_{i}
$$

In order to model the behavior of the selfish mobile nodes, we introduce the concept of best response and Nash Equilibrium.

The best response of player $i$ to the profile $s_{-i}$ is a strategy such that:

$$
B_{i}\left(s_{-i}\right)=\arg \max _{s_{i} \in S_{i}} \pi_{i}\left(s_{i}, s_{-i}\right)
$$

If player $i$ plays strategy $B_{i}\left(s_{-i}\right)$, it reaches the maximum from the obtainable payoffs given that the other players play $s_{-i}$.

The pure-strategy profile $s^{*}$ is a Nash Equilibrium if the following equation holds for $s^{*}$ :

$$
s_{i}^{*}=B_{i}\left(s_{-i}^{*}\right), \forall i \in P
$$

Namely, in Nash Equilibria none of the players can increase their payoff by changing their strategy unilaterally.

A game $G=\left[P,\left\{S_{i}\right\},\left\{\pi_{i}()\right\}\right]$ is symmetric if each player has the same strategy space $\left(S_{0}=S_{1}=\ldots=S\right)$ and their payoff functions are equal $\left(\pi_{i}\left(s_{i}, s_{-i}\right)=\pi_{j}\left(s_{j}, s_{-j}\right)\right.$ for $s_{i}=$ $s_{j}$ and $s_{-i}=s_{-j}$, where $i, j \in P$ ). A symmetric game $G$ can be denoted by $[P, S, \pi()]$.

As one can see, the barter game is a symmetric game, because the strategy space defined in the game is identical for all players. In our system model, the nodes are not distinguished. Thus, they can maximize their payoff in the 
same way and they get the same payoff in the same strategy profile.

In the analysis of the barter mechanism, we are looking for the Nash Equilibria. We limited ourselves to find only pure-strategy, symmetric Nash Equilibria. This is because, we assumed that each mobile node is a player, which leads to the analysis of a game with a $|P|$-dimensional strategy space. The exhaustive analysis of the entire strategy space is thus infeasible by means of simulations.

A symmetric game has symmetric pure-strategy equilibria [8], if the strategy space is a nonempty, convex and compact subset of some Euclidean space while the function of payoff is continuous in the strategy and quasiconcave. In our case, the strategy space is the interval $[0,1]$, which corresponds to the conditions of existing symmetric purestrategy equilibrium. Whereas, the properties of the payoff function are not verifiable, the results of the simulations will show that the conditions hold.

If we expand (8) and (9) according to the symmetric game and equilibrium, $\left\{s^{*}\right\}$ is Nash Equilibrium if the following equation holds for any player $i \in P$ :

$$
\begin{aligned}
& s_{i}^{*}=\arg \max _{s_{i} \in S} \pi\left(s_{0}^{*}, s_{1}^{*}, \ldots, s_{i}, \ldots\right), \\
& \text { where } s_{u}^{*}=s_{v}^{*} \forall u, v \in P /\{i\}
\end{aligned}
$$

As one can see, it is easy to verify that a specific strategy profile $\left\{s^{\prime}\right\}$ is a Nash Equilibrium or not. Considering any player $i \in P$ - without loss of generality $i=0$, called player null - if it is worth for player $i$ to deviate, $\left\{s^{\prime}\right\}$ is not a Nash Equilibrium, whereas if $s^{\prime}$ is the best response to player $i$ then $s^{\prime}$ will be the best response strategy for all the other players too, as the players have equal payoff functions.

Therefore, to find the symmetric pure-strategy Nash Equilibria, it is not necessary to examine the whole $|P|$ dimensional strategy space, but investigation of a twodimensional space is enough. In order to find all the symmetric pure-strategy Nash Equilibria, we consider all the symmetric pure-strategies $\left\{s^{\prime}\right\}$ as Nash Equilibria candidates. Then, we consider the whole strategy space that player null can play to check if a Nash Equilibrium candidate is indeed a Nash Equilibirium or not. The strategy space which is required to be analyzed to find all the symmetric pure-strategy Nash Equilibria can be seen in Eq. (11).

$$
\left\{s, s^{\prime}, \ldots, s^{\prime}\right\}, \forall s \in S, \text { and } \forall s^{\prime} \in S
$$

Thus, due to the symmetry of the game, the analysis is independent of the number of players.

\section{Results}

We run simulations to analyze the efficiency of the barter mechanism as we did in Section 2. The simulations were executed with the same parameters such that we can compare the barter based mechanism to the other two analyzed cases: 1) when the messages disseminate ideally (this case gives an upperbound for the goodput of the network), and 2) when the nodes download only primary messages.

As we have already described, the mobile nodes do not change their strategy during a game. Therefore, in each simulation run, the mobile nodes play a predefined strategy chosen from discrete values of the strategy space. The discrete values are the values from 0 to 1 increasing by 0.05 .

We run a simulation with a concrete parameter set six times, and we consider the average goodput of player null. The obtained goodput of the other mobile nodes is irrelevant, because the game is analyzed from one, representative player's point of view according to the description in Section 4

Due to the above described discretization, each mobile node's strategy can take 21 possible values. This means that we had to run $21^{2}=441$ simulations for each parameter setting in order to find the pure-strategy, symmetric Nash Equilibria. The best response function of some parameter settings can be seen in Figures 5(a) and 5(b).

In Figure 5, on the vertical axis, there are the strategies that player null can choose, while on the horizontal axis, the strategy space of the other players is placed. The Nash Equilibrium candidates are the strategy profiles where player null and the other players choose the same strategy; these are denoted by solid, black points in Figure 5 . Whereas, the best response strategy of player null to a specific strategy profile of the other players is denoted by empty circles. Figure 5(a) shows the result of a simulation set where the messages devaluate according to the function $\delta_{0}$ (see Eq. (1)) the popularity of the generated messages is 0.4 and mobile nodes move according to the restricted random waypoint model. In this parameter set, the player null can get the highest payoff if its strategy is 0.15 independently from other player's strategy. According to this, the Nash Equilibrium is the strategy set where all the nodes play with strategy 0.15 . In other simulation sets the best response strategy value in the most cases is independent of the other players' strategy, but the value of the best response is different. To give an overview of the value of player null's best response in all simulation sets, we plotted a histogram in Figure 8.

In Figures 6(a) and 6(b), the results of simulations are plotted in an extended form. In these figures the payoff of player null is plotted against the strategies of player null and other players. The best response strategy of player null is the strategy where the payoff of the player null is maximal given a fixed strategy of the other players. The best response strategy is denoted by big black circles in Figure 6 .

As one can see, the payoff of player null intensively falls down if player null does not cooperate $(s=0)$. The nodes are encouraged to carry messages when the barter mechanism is used, because their goodput is higher if they do so (even if they are not directly interested in those messages). The payoff of player null intensively falls down too, if it is too altruistic $(s=1)$, namely if it values the secondary messages as high as their primary messages. It helps the other mobile nodes, but it misses to obtain messages that 


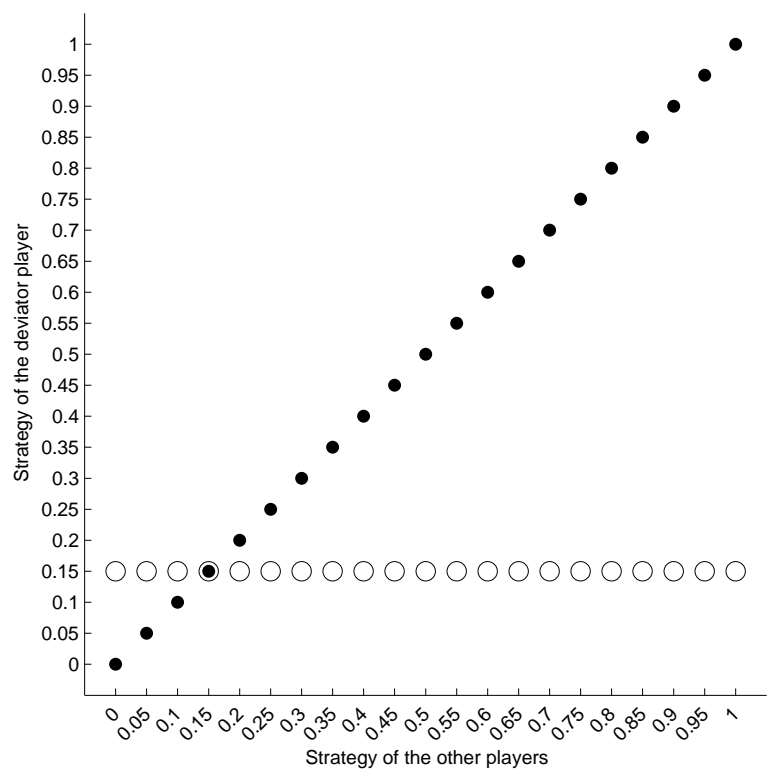

(a) RRW

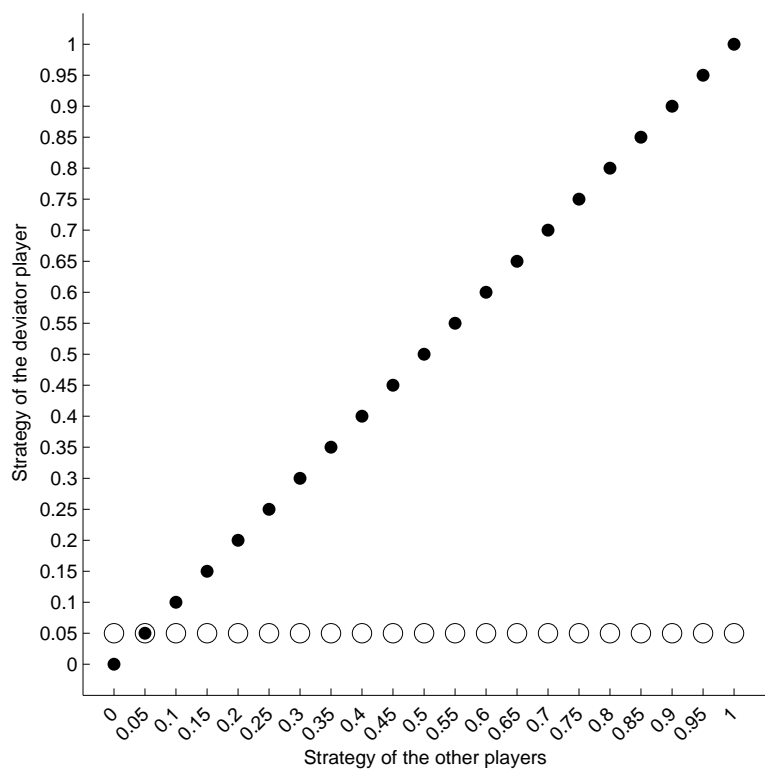

(b) SUMO

Fig. 5. Best response: Nash Equilibrium candidates are denoted by solid, black points, while empty circles show the best response strategy of player null. The Nash Equilibria are the strategy profiles where the best response function meets the Nash Equilibrium candidates.

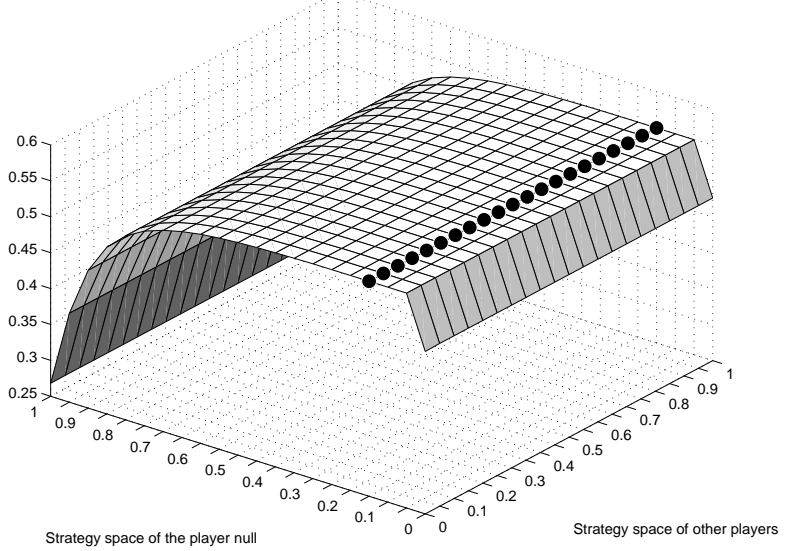

(a) RRW

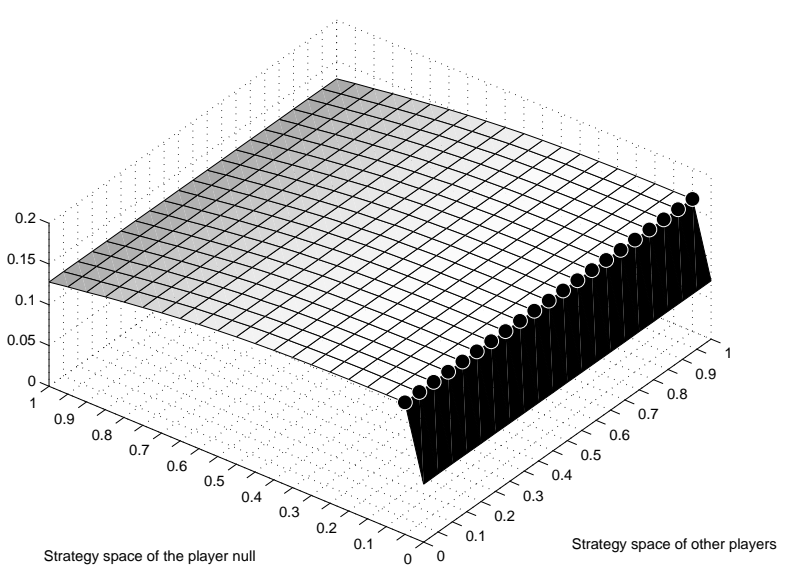

(b) SUMO

Fig. 6. Gain of player null: The gain (goodput) of player null is plotted against the strategy of player null and other players. The best response function of player null is denoted by big black circles.

it is interested in and suffer from goodput decrease.

To understand the reasons, we created some statistics during the simulations concerning the number and the type of message exchanges. In Figure 7(a), we plotted the number of all message exchanges against the strategies of player null and other players, and also classified the downloads by the type of the downloaded message (primary or secondary), these are plotted in Figure 7(b) and 7(c), respectively.

The Figure 7(a) shows that the message exchange significantly decreases when the mobile nodes do not cooperate at all $(s=0)$. As the message exchange decreases, the messages disseminate slower and the mobile nodes suffer from decreasing goodput.
However, the mobile nodes also reach lower goodput if they are too altruistic. The reason is the following: As one can see in Figure 7, when a player increases its secondary/primary value, the number of obtained primary messages decreases while the number of obtained secondary message increases, whereas the number of message exchange does not vary appreciably (not taking into account when the mobile nodes do not cooperate at all). This shows that the mobile nodes following altruistic strategy do not utilize the investment of downloading secondary messages, but download more secondary ones.

To conclude the result of simulations, we can state that in the simulated cases, the strategy which is most beneficial individually - the Nash Equilibirium of the barter game - 


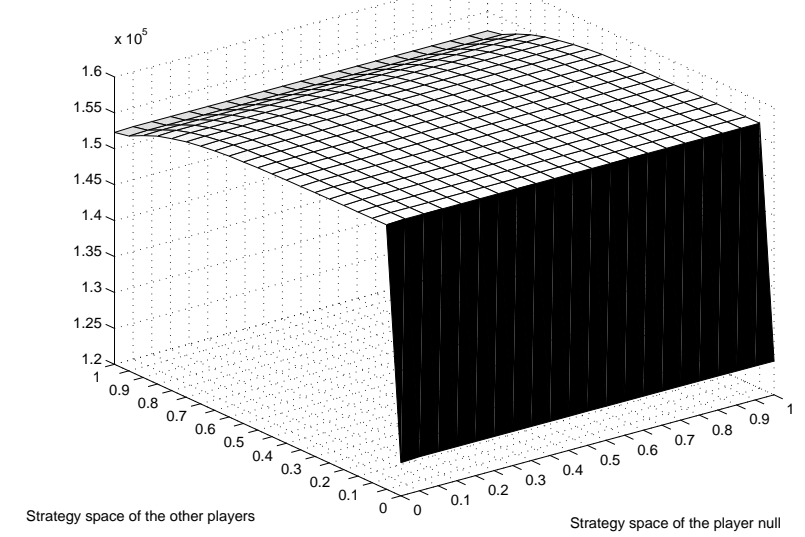

(a) Number of message exchange

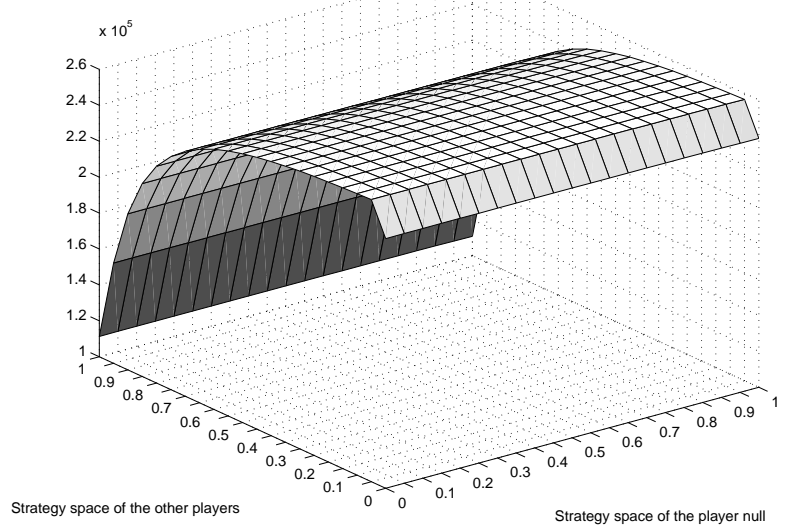

(b) Number of primary message download

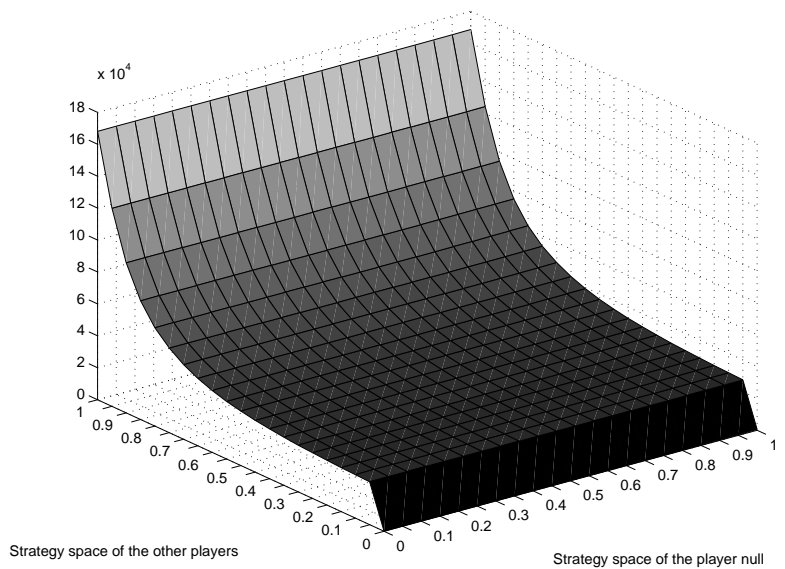

(c) Number of secondary message download

Fig. 7. Message download statistics: Number of message exchange, primary and secondary message download is plotted against different strategy profiles

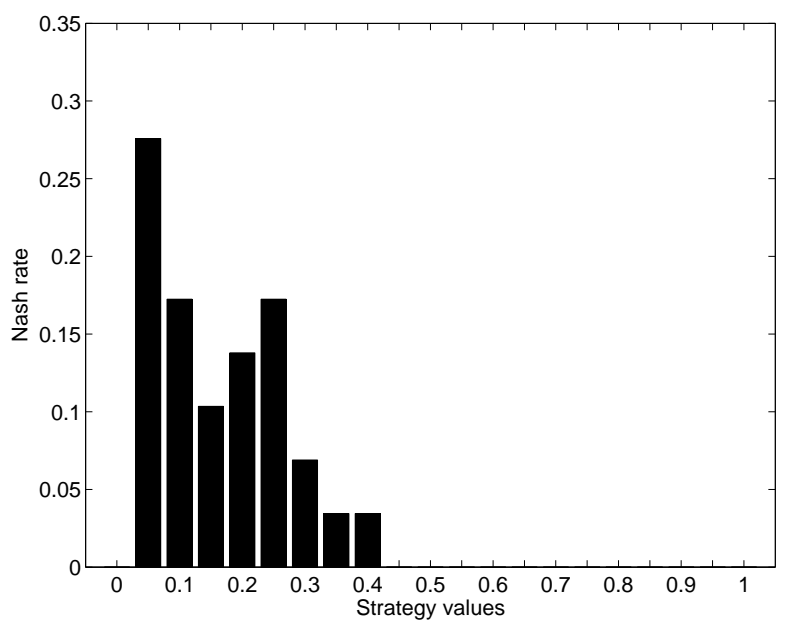

(a) RRW

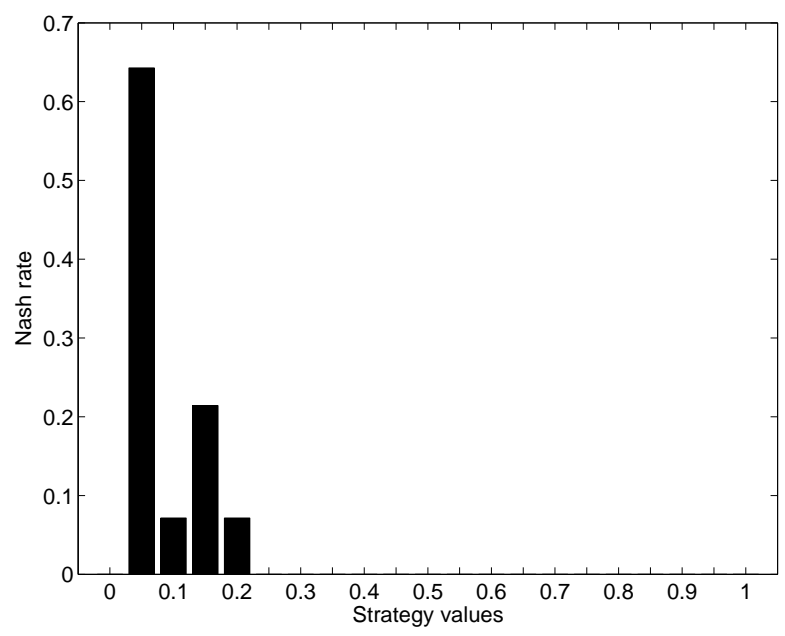

(b) SUMO

Fig. 8. Histogram of Nash Equilibrium values

to set the secondary/primary ratio to a low value but not to 0 . Therefore, it is beneficial to help the other nodes $(s \neq 0)$ carrying their messages when the nodes exchange messages only in fair manner. However, if they are too altruistic, they download primary messages with less probability, and their goodput decreases. This can be seen is Figure 8, where the histogram of the Nash Equilibrium strategy values is plotted. The Nash Equilibrium values are obtained from all 


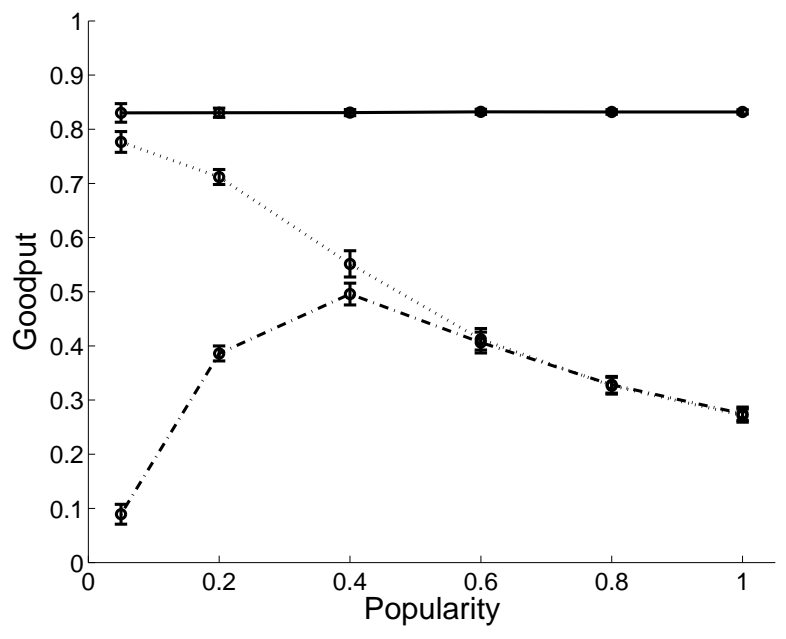

(a) RRW

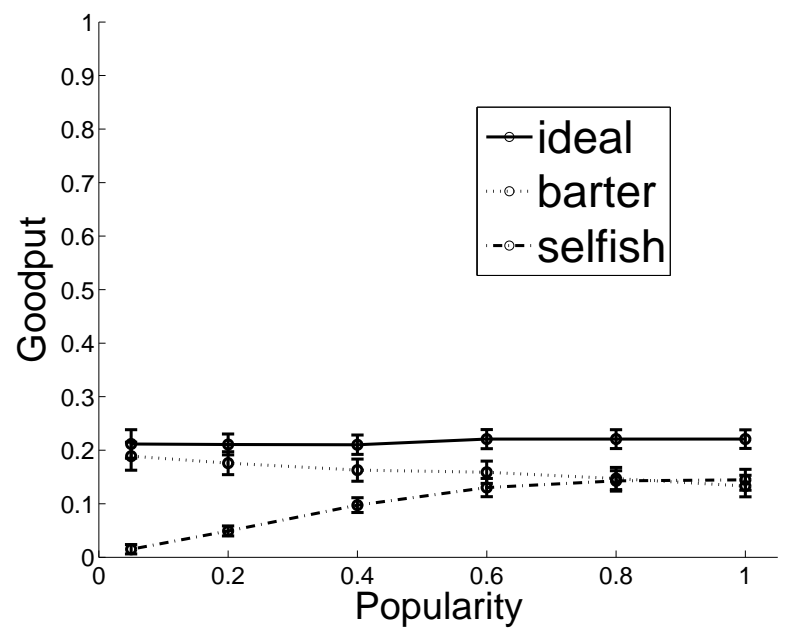

(b) SUMO

Fig. 9. Goodput with barter mechanism: The steady state goodput plotted against the popularity value of generated messages as done in Figure 4. The goodput values of barter mechanism are obtained in the Nash Equilibrium. The bars show the $95 \%$ confidence intervals.

the simulation sets and grouped by the mobility models.

In Figures 9(a) and 9(b), the network goodput is plotted against the popularity attribute of the generated messages with restricted random waypoint and SUMO mobility model, respectively. It was done also in Figure 4, but these figures are supplemented with the goodput in Nash Equilibrium of the barter game. As it can be seen, the barter mechanism eliminated the selfish carrier effect, i.e. increases the goodput in the networks where the popularity value of generated messages is low. Furthermore, the goodput is as high as the optimal goodput. Meanwhile, the barter mechanism does not decrease the goodput when the message popularity is high which is affected by the implicit cost. The implicit cost is a system property, therefore it cannot be compensated.

In Figure 10, the effect of different delete threshold values $(D=0.05,0.2,0.5,0.75,0.95)$ are analyzed in the restricted random waypoint model. Note that mobile nodes delete a message from their memory when its value goes below D. In Figure 10(a), we show which strategies are Nash Equilibrium strategies using different $D$ values. One can see that the set of Nash Equilibria increases with increasing $D$ value. The reason is the following: as the $D$ value increases the mobile nodes delete the messages earlier and therefore, they store less messages in their memory on average. For this reason, the effect of the $S P$ ratio decreases as the variety of the messages decreases. Thus, more and more strategies result in the same goodput value.

In Figure 10(b), the goodput value is plotted against the $D$ values. As it is expected, the goodput value decreases with increasing $D$ value, because the mobile nodes delete the messages earlier. This has a doubled effect in the case of the barter: 1) A mobile node may not be able to obtain a message, and 2) A mobile node may not be able to offer any messages, thus, it can not obtain other messages.

\section{Related work}

So far, the problem of selfish nodes has been addressed mainly in the context of mobile ad hoc networks. The proposed solutions to stimulate cooperation can be broadly classified into two categories: reputation systems and virtual payment based methods. Several researchers proposed reputation systems for ad hoc networks [9,10], and in [11], an opportunistic solution is presented. For the virtual payment based methods, some proposed solutions can found in $[12,13]$ in traditional ad hoc networks and there are opportunistic network specific solutions in [14]. Usually, these solutions require authentication (and related key management), and/or the presence of a trusted third party. In addition, the payment based solution also raises the problem of determining the price of different actions (see e.g., [15]).

Researchers have also studied under what conditions cooperation can emerge spontaneously among the nodes in ad hoc networks (see e.g., [16,17]).

The application of delay-tolerant networks for personal wireless communications is considered in [18]. In particular, the authors show, by analytical tools and by means of simulations, that delay-tolerant networks can achieve a reasonably high throughput such that they can support various personal communication services.

In [1], the authors raise the problem of selfishness in delay tolerant networks. The authors study the performance of three representative routing algorithms in the presence of some selfish nodes. They show that when the nodes behave selfishly, the performance decreases, in the sense that messages are delivered with a longer delay if they are delivered at all. However, the authors do not propose any mechanism to stimulate cooperation. The results presented in [1], can be viewed as a motivation for our work.

In [19], the authors considered the same subject. They have proven by analytical tools that the most beneficial 


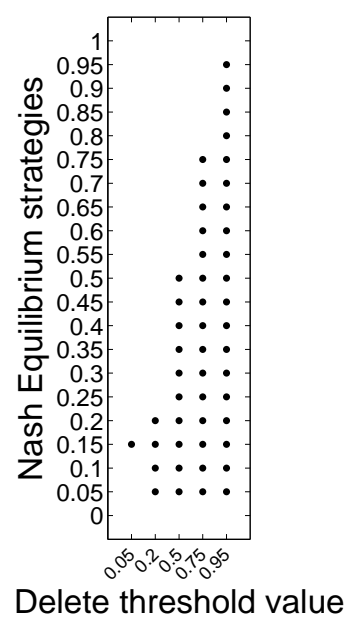

(a) Nash Equilibria against different delete threshold values

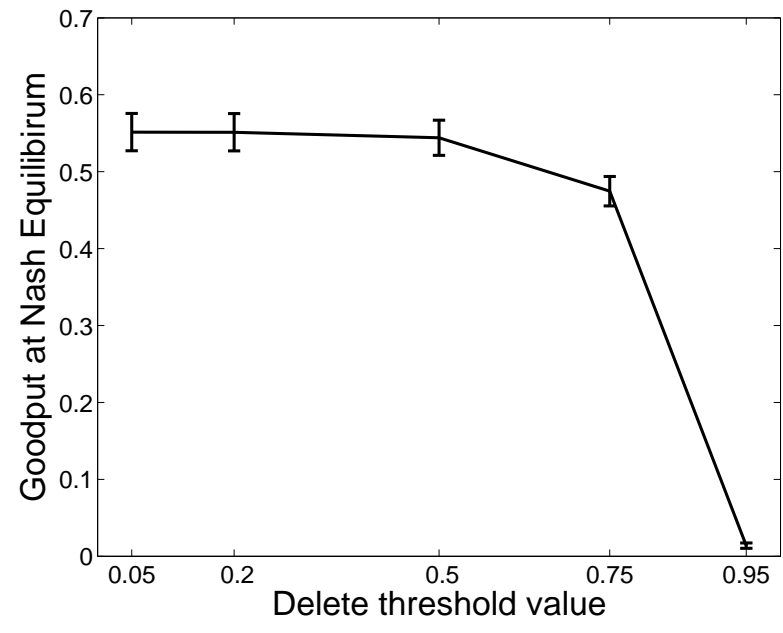

(b) Payoff against different delete threshold values

Fig. 10. Analysis on the effect of different delete threshold values. The bars show the $95 \%$ confidence intervals.

behavior is to follow a forwarding strategy that the mobile nodes agreed on before. The forwarding strategy is described by the probability of forwarding uninterested messages. In contrast to the above mentioned analytical model, we investigate a more complex model.

The barter mechanism was introduced first and analyzed by a preliminary model in [2]. As explained in the Introduction, we have rebuilt the system and the game model and we extended the simulation sets, too. The most important new contributions are that we pin-pointed the scenarios where encouraging mechanism is required and we showed that the barter based mechanism increases data delivery in a more general and realistic model. Furthermore, we presented a more exhaustive analysis of the results.

\section{Conclusion}

In opportunistic networks, selfish nodes can exploit the services provided by other nodes by downloading messages that interest them, but refusing to store and distribute messages for the benefit of other nodes. To eliminate the harmful influence of selfish behavior, we proposed a mechanism which is based on the principles of barter. The users trade in messages, meaning that they can download a message from another user if they also provide a message in return. We analyzed our proposed solution using a game-theoretic framework, and showed that it indeed discourages selfishness. More precisely, the analysis shows that it is worth for users collecting, carrying and disseminating messages even if they are not interested in them, which has a positive effect on quality of data dissemination. In particular, the results show that, in realistic scenarios, the message delivery rate considerably increases if the mobile nodes follow the Nash Equilibrium strategy in the barter mechanism compared to the data dissemination protocol when no encouraging mechanism is present.

\section{Acknowledgements}

The work presented in this paper has been partially supported by the BIONETS Project (www.bionets.org) and the Hungarian Scientific Research Funds (OTKA T046664).

\section{References}

[1] A. V. Antonis Panagakis, I. Stavrakakis, On the Effects of Cooperation in DTNs, in: Proc. of The Second IEEE/CreateNet/ICST International Conference on COMmunication System softWAre and MiddlewaRE (COMSWARE), 2007, pp. 1-6.

[2] L. Buttyán, L. Dóra, M. Félegyházi, I. Vajda, Barter-based cooperation in delay-tolerant personal wireless networks, in: In Proceedings of the First IEEE WoWMoM Workshop on Autonomic and Opportunistic Communications, IEEE Computer Society Press, 2007, pp. 1-6.

[3] SUMO - Simulation of Urban MObility, http://sumo.sourceforge.net/.

[4] M. Félegyházi, J.-P. Hubaux, Game Theory in Wireless Networks: A Tutorial, Tech. Rep. LCA-REPORT-2006-002, EPFL (Feb. 2006).

[5] D. Fudenberg, J. Tirole, Game Theory, MIT Press, 1991.

[6] R. Gibbons, A Primer in Game Theory, Prentice Hall, 1992.

[7] M. J. Osborne, A. Rubinstein, A Course in Game Theory, The MIT Press, Cambridge, MA, 1994.

[8] S.-F. Cheng, D. M. Reeves, Y. Vorobeychik, M. P. Wellman, Notes on Equilibria in Symmetric Games, in: In Proceedings of Workshop on Game Theory and Decision Theory, 2004.

[9] P. Michiardi, R. Molva, Core: A COllaborative REputation mechanism to enforce node cooperation in Mobile Ad Hoc Networks, in: Proc. of Communication and Multimedia Security 2002, 2002, pp. 107-121.

[10] S. Buchegger, J.-Y. L. Boudec, Performance Analysis of the CONFIDANT Protocol (Cooperation Of Nodes-Fairness In Dynamic Ad-Hoc NeTworks), in: Proc. of the 3rd ACM International Symposium on Mobile Ad Hoc Networking and Computing (MobiHoc'02), 2002, pp. 80-91.

[11] M. Voss, A. Heinemann, M. ühlhäuser, A Privacy Preserving Reputation System for Mobile Information Dissemination 
Networks, in: First International Conference on Security and Privacy for Emerging Areas in Communications Networks (SECURECOMM'05), IEEE, 2005, pp. 171-181.

[12] L. Buttyán, J.-P. Hubaux, Stimulating Cooperation in SelfOrganizing Mobile Ad Hoc Networks, ACM/Kluwer Mobile Networks and Applications (MONET) Special Issue on Mobile Ad Hoc Networks 8 (5), pp. 579-592.

[13] S. Zhong, Y. R. Yang, J. Chen, Sprite: A Simple, CheatProof, Credit-Based System for Mobile Ad Hoc Networks, in: Proceedings of the IEEE Conference on Computer Communications (INFOCOM '03), 2003.

[14] M. Önen, A. Shikfa, R. Molva, Optimistic fair exchange for secure forwarding, in: SPEUCS 2007, 1st Workshop on the Security and Privacy of Emerging Ubiquitous Communication Systems, 2007, pp. 1-5.

[15] J. Crowcroft, R. Gibbens, F. Kelly, S. Östring, Modelling incentives for collaboration in mobile ad hoc networks, Perform. Eval. 57 (4) (2004) 427-439.

[16] V. Srinivasan, P. Nuggehalli, C. F. Chiasserini, R. R. Rao, Cooperation in Wireless Ad Hoc Networks, in: Proceedings of the IEEE Conference on Computer Communications (INFOCOM '03), 2003, pp. 808-817.

[17] M. Félegyházi, J.-P. Hubaux, L. Buttyán, Nash Equilibria of Packet Forwarding Strategies in Wireless Ad Hoc Networks, IEEE Transactions on Mobile Computing 5 (5).

[18] G. Karlsson, V. Lenders, M. May, Delay-tolerant broadcasting, in: CHANTS '06: Proceedings of the 2006 SIGCOMM workshop on Challenged networks, ACM Press, New York, NY, USA, 2006, pp. 197-204.

[19] I. Koukoutsidis, E. Jaho, I. Stavrakakis, Cooperative content retrieval in nomadic sensor networks, in: Infocom MOVE Workshop 2008 (MObile Networking for Vehicular Environments), IEEE, 2008.

\section{Appendix A. Convergence of the goodput}

In this section, we prove that the goodput of the nodes converges to a limiting value. This can also be seen in Figure A.1 where the goodput of some randomly chosen mobile nodes is plotted against the time. In Figure A.2, the average goodput and its dispersion of all mobile nodes is plotted against the time. After this analysis, we can state that the goodput obtained after a fixed-number of time steps in simulation close to the steady-state goodput.

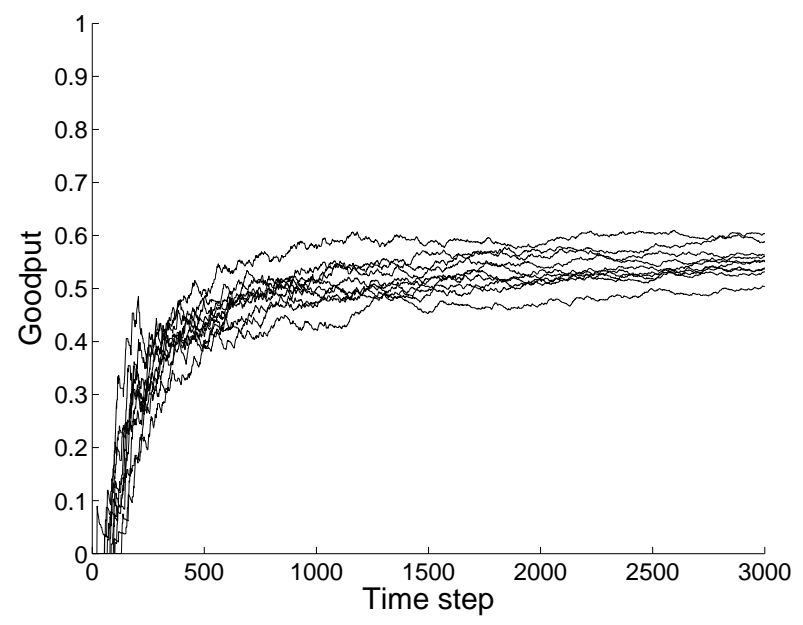

Fig. A.1. The convergence of the goodput of some sample nodes

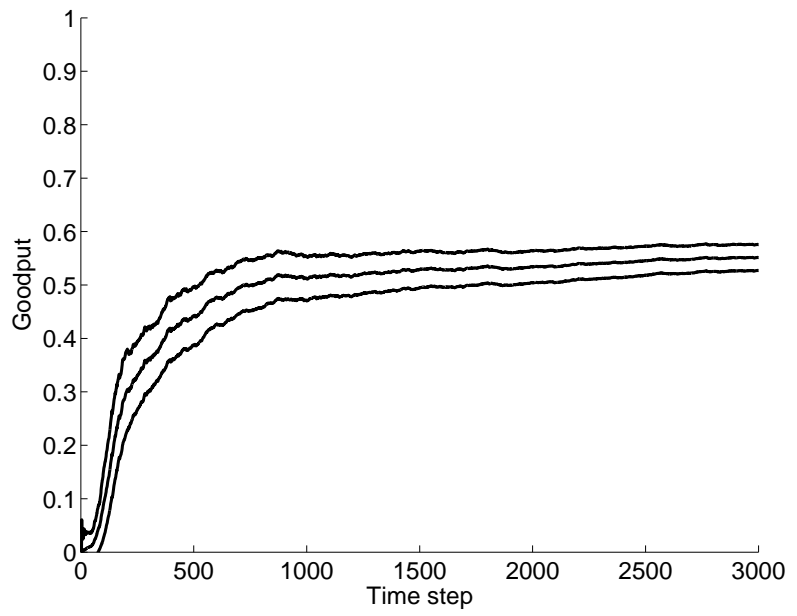

Fig. A.2. The convergence of the average goodput and its dispersion

The state of the system described in Section 2 at time $t$ is

$$
\begin{aligned}
s(t)=\{ & B_{1}(t), B_{2}(t), \ldots, B_{N}(t), \\
& Z_{1}(t), Z_{2}(t), \ldots, Z_{N}(t), \\
& \left.H_{1}(t), H_{2}(t), \ldots, H_{N}(t)\right\}
\end{aligned}
$$

where

- $N$ is the number of nodes

- $B_{i}(t)=\left[m_{i_{1}}, m_{i_{2}}, \ldots\right]$ is the buffer of node $i$, where the messages are stored.

- $Z_{i}(t) \in\{*, m\}$ is message stored in the memory of the message node $i$, where $*$ denotes the case when no message is stored at time t, otherwise $m$ stands for the generated message, which arrives from the - in principle infinite space of messages.

- $H_{i}(t)$ is the position of node $i$ on the field $F$.

We consider a finite state Markovian model in what follows.

Note, that the state space can be described by a deterministic mapping:

$$
\begin{aligned}
s(t+1)=F[ & s(t), \\
& r_{1}(t+1), r_{2}(t+1), \ldots, r_{N}(t+1), \\
& r_{1}^{\prime}(t+1), r_{2}^{\prime}(t+1), \ldots, r_{n}^{\prime}(t+1), \\
& \left.r_{1}^{\prime \prime}(t+1), r_{2}^{\prime \prime}(t+1), \ldots, r_{M}^{\prime \prime}(t+1)\right]
\end{aligned}
$$

where

- $r_{i}(t+1)$ is a random element used as an input by the algorithm to calculate the next step of node $i(1 \leq i \leq$ $N$ ) on field $F$ at time $t+1$

- $r_{i}^{\prime}(t+1)$ is a random element used as an input of message generation of message node $i(1 \leq i \leq n)$ at time $t+1$.

- $r_{i}^{\prime \prime}(t+1)$ is a random element used as an input of the node pairing in meeting point $i(1 \leq i \leq M)$.

The random numbers are generated independently of the time.

Note, that the state transition mapping is time independent. The sequence of state random variables $S(0), S(1), \ldots, S(t), \ldots$ constitutes a discrete time homogenous Markovian chain. The transition matrix of the Markovian process can be derived from (A.1) and (A.2). 
As one can see the state space of the Markovian model described above is infinite, however with some feasible assumptions the model can be converted to a finite state model.

- Note that the memory of message nodes was assumed to be unlimited in the whole paper, however an upperbound can be defined. Recall that the mobile nodes delete the messages if the message is older than $T$ time step. Let the number of message nodes be $n$. The greatest number of messages is generated if all the message nodes generate a new message in each time step. A message disappear from the system after $T$ time steps. Therefore, the greatest number of messages that a node may store is $L=n \cdot T$. Hereby, $B_{i}(t)=\left[m_{i_{1}}, m_{i_{2}}, \ldots, m_{i_{L}}\right]$.

- In the Markovian model described above, the $m$ messages arrive from infinite space as there was no restriction for it. However, it is feasible to assume that the length of the digital contents that the nodes exchange is limited, let us assume to be $l$. In that case, the size of the message space is $2^{l}$.

A Markovian chain is ergodic, if the following limiting value exist:

$$
\lim _{n \rightarrow \infty} P_{i k}^{(n)}=P_{k}
$$

these are independent of $i$ and

$$
\sum_{k=1}^{\infty} P_{k}=1
$$

As the classic theorem of Markovian chains claims, a finite state homogenous Markovian chain is ergodic, if it is irreducible and aperiodic. Particularly, there is a time step $t$ and a state $j$, such that state $j$ can be reached from arbitrary initial state $i$ with positive probability with time step $t$. The convergence to limiting distribution $P_{j}$ is exponential, which means the following: let $P_{i j}^{(t)}$ denote the probability, that the Markovian chain starting from state $i$ arrives at state $j$ with $t$ steps, furthermore let denote the stationary probability of state $j$, the difference $\left|P_{i j}^{(t)}-P_{j}\right|$ decreases exponentially when $t$ tends to infinity (Theorem of Markov). In this case, uniform exponential bound exists for difference $\left|P_{i j}^{(t)}-P_{j}\right|$ independently of $j$.

In our model, the proof of the condition for ergodicity is the following: Assume the system is in an arbitrary state. We select a state $k$, let this state be the following, the buffer of the first node contains a single fresh message, while all other buffers are empty. Such a state can be produced the following way: First we empty all the buffers: the users move or stagnate at a fixed position such a way they escape meeting message sources. As the time passes the aging messages drop out from the buffer. Then the first node approaches a message source where it receives a message.

As it is shown above, our system is ergodic. The distribution of the stationary state is approached at exponential rate.

The goodput of a node until time step $t$ - as it is already described in (5) - is:

$$
G_{i}(t)=\frac{\sum_{t_{j}=0}^{t} v_{i}\left(t_{j}\right)}{\sum_{t_{j}=0}^{t} M_{i}^{p}\left(t_{j}\right)}
$$

where the $v_{i}(t)$ is the gain that node $i$ received in time step $t$, and $M_{i}^{p}(t)$ is the number of primary messages of node $i$ generated in time step $t$.

As one can see, the goodput is affected by the transient state of the system also, not just on the stationary state. However, from the ergodicity of the Markovian chain, it follows that the effect of the transient state become negligible and fades away with exponential rate if the time goes to infinity. By empirical observation, it is appropriate to consider the goodput after time step 3000 and the goodput will not change in the future considerably.

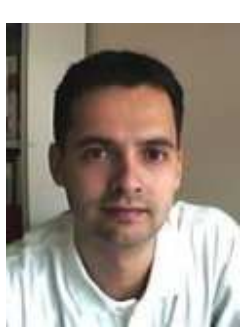

Levente Buttyán received the M.Sc. degree in Computer Science from the Budapest University of Technology and Economics (BME) in 1995, and the Ph.D. degree from the Swiss Federal Institute of Technology, Lausanne (EPFL) in 2002. In 2003, he joined the Department of Telecommunications at BME, where he currently holds a position as Associate Professor and works in the Laboratory of Cryptography and Systems Security (CrySyS). His research interests are in the design and analysis of security protocols for wired and wireless networks, including wireless sensor networks and ad hoc networks. More information is available at http://www.hit.bme.hu/ buttyan/

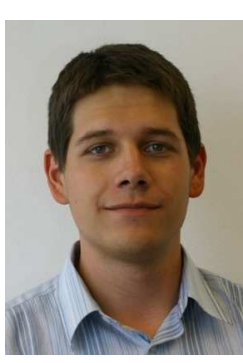

László Dóra received the M.Sc. degree in Computer Science from the Budapest University of Technology and Economics (BME) in 2005. During his M.Sc. he joined the Laboratory of Cryptography and Systems Security (CrySyS) in 2004. Since 2005 he is a Ph.D. student at the same laboratory under the supervision of Levente Buttyán. His research interests are in security of opportunistic and mesh networks. More information is available at http://www.crysys.hu/members/ldora/ 
Márk Félegyházi was born in Budapest, Hungary in 1978. He holds an M.Sc. from Budapest University of Technology and Economics, Hungary (2001) and a Ph.D. from EPFL, Switzerland (2007). His research focuses on designing incentive mechanisms for computer networks. Currently, at UC Berkeley, he is interested in incentive design to improve the security of com-

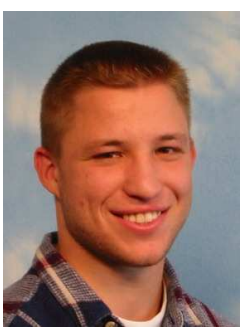
puter systems, notably to increase user awareness, to manage risks and liabilities and to design practical reputation systems. During his stay at EPFL, he worked on incentives for wireless social community networks, on reputation systems based on game theory to avoid denial of service in wireless ad hoc networks and on incentive-aware network design for various wireless networks. His earlier work at Ericsson Research, Hungary included Mobile IP protocol testbed implementation and the design of ad hoc network protocols for the Bluetooth short-range wireless technology.

István Vajda is a Professor at the Department of Telecommunications, Budapest University of Technology and Economics (BME). He is the Head of the Laboratory of Cryptography and Systems Security (CrySyS). His research interests are in Cryptography and Coding Theory. He has teaching experience in Algebraic Coding Theory, Cryptog-

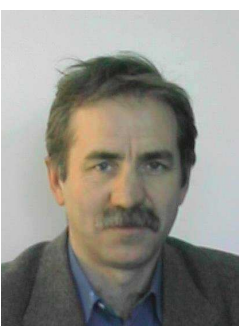
raphy, and Information Theory. 